\title{
Virtual-work-based Optimization Design on Compliant Transmission Mechanism for Flapping-wing Aerial Vehicles
}

\author{
Chao Zhang, Claudio Rossi
}

\author{
Wei He
}

\author{
Julian Colorado
}

\begin{abstract}
This paper presents a method for analysing and optimizing the design of a compliant transmission mechanism for a flapping-wing aerial vehicle. Its purpose is of minimizing the peak input torque required from a driving motor. In order to maintain the stability of flight, minimizing the peak input torque is necessary. To this purpose, first, a pseudo-rigid-body model was built and a kinematic analysis of the model was carried out. Next, the aerodynamic torque generated by flapping wings was calculated. Then, the input torque required to keep the flight of the vehicle was solved by using the principle of virtual work. The values of the primary attributes at compliant joints (i.e., the torsional stiffness of virtual spring and the initial neutral angular position) were optimized. By comparing to a full rigidbody mechanism, the compliant transmission mechanism with well-optimized parameters can reduce the peak input torque up to $66.0 \%$.
\end{abstract}

\section{INTRODUCTION}

In recent years, natural flying insects, birds and bats are becoming attractive models to imitate in design and development of flapping-wing aerial vehicles (FWAVs) as they are capable of excellent maneuverability. In such case, designing a proper and effective flapping mechanism plays a significant role in fabricating a FWAV. During every flapping cycle, wings have to experience an acceleration phase from rest at the start of a stroke and a deceleration phase to stop at the end of the stroke and then the process reverses in the next stroke, which can easily get shocks to the driving motor and even brake it, due to the effect of aerodynamic the forces produced by flapping wings. These shocks needs to be minimized in the design of flapping mechanism as much as possible for making sure that the motor safely run and thereby generate stable lift and thrust forces required.

The introduction of coil springs can mitigate these unexpected shocks [1] in bio-inspired robots. Research results from both Madangopal et al. [2] and Baek et al. [3] show that the introduction of linear coil springs can minimize the required input peak torque or input power. The use of linear coil springs which are directly connected to DC motors in direct-driving flapping-flight prototypes can also be found in the literature [4], [5]. However, additional springs not only bring unneeded payloads, but also do not reduce joint friction energy losses [6]. Recently, piezoelectric or electromagnetic actuators are also introduced to couple with the mechanism for driving flapping wings toward resonance [4], [7]-[11]. However, neither piezoelectric nor electromagnetic actuators are suitable for systems with a higher desired payload due to their high power requirements and limits in displacement and forces [4].

Compared to the above spring mechanisms and piezoelectric or electromagnetic actuators, compliant mechanisms seem to be more attractive solutions. Such mechanisms are multifunctional structures which combine functions of mechanical parts with elastic energy-stored components together [12]. Moreover, compliant mechanisms have advantages compared to rigid-body mechanisms, such as lower wear, friction, and backlash [13], [14], and are compatibility with microelectromechanical systems (MEMS) processing techniques [15].

Compliant mechanisms for FWAVs have been already employed in few prototypes. In [16], a thorax-like clicking compliant mechanism inspired by Dipteran insects is proposed. This research showed that clicking compliant mechanisms could produce more thrust per input power than a conventional rigid-body counterpart. Finally, Sahai et al [17] demonstrated that flapping transmission with rubber flexural hinges can reduce input power up to $20 \%$.

In the literature, few works that introduce compliant mechanisms into FWAVs, take into account the influences of the characteristics of compliant joints or hinges (like the stiffnesses of virtual spring and the neutral angular positions) on the final input peak torque. In addition, most works lack an exhaustive theoretical analysis. In this paper, we present an elaborate theoretical analysis on the transmission mechanism and the effects of the compliant attributes on the final peak input torque by using the principle of virtual work.

In the following, we describe an analysis of a representative compliant driving mechanism and use a virtual-work based method to explore the influence of compliant attributes on the 


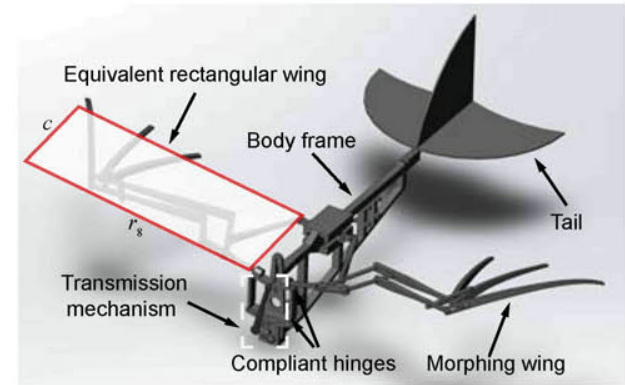

Fig. 1. A closeup of the CAD model for a bat-inspired flapping-wing aerial vehicle.

TABLE I

COMPONENT DIMENSIONS OF THE COMPLIANT TRANSMISSION MECHANiSM (SEE ALSO FIG. 2).

\begin{tabular}{c||c||c}
\hline \multicolumn{1}{c||}{ Link } & Symbol & Scale \\
\hline \hline $\mathrm{OA}$ & $r_{1}$ & $6.0 \mathrm{~mm}$ \\
\hline $\mathrm{AB}$ & $r_{2}$ & $32.8 \mathrm{~mm}$ \\
\hline $\mathrm{BC}$ & $r_{3}$ & $15.0 \mathrm{~mm}$ \\
\hline $\mathrm{CD}$ & $r_{4}$ & $22.4 \mathrm{~mm}$ \\
\hline OB & $r_{5}$ & - \\
\hline Horizontal Distance OD & $r_{6}$ & $15.0 \mathrm{~mm}$ \\
\hline Vertical Distance OD & $r_{7}$ & $13.0 \mathrm{~mm}$ \\
\hline CE & $r_{8}$ & $235.0 \mathrm{~mm}$ \\
\hline
\end{tabular}

input torque. Additionally, the optimization of the compliant joints is carried out.

\section{KinEMATICS OF FLAPPING TRANSMISSION MECHANISM WITH COMPLIANT JOINTS}

In this section, we present a flapping transmission mechanism with small-length lightweight compliant hinges and then perform kinematic analysis of it. The proposed compliant hinges are equivalent as revolute joints with virtual springs based on the pseudo-rigid-body (PRB) model theory of compliant mechanisms proposed by L. L. Howell in [18]. A CAD cutaway of main airframes is shown in Fig. 1. The robot consists of morphing wings, a tail, a body frame and a compliant transmission mechanism. A small DC motor running in anti-clockwise direction is employed to drive a compliant transmission mechanism to realize flapping motions. The mass of wing frames is assumed to be uniformly distributed along the length of the wing spar. Since the robot has a symmetrical structure, only half of the structure is analysed here. The schematic closeup of the half transmission mechanism is shown in Fig. 2(a) and its corresponding PRB model is presented in Fig. 2(b). The dimensions of the primary frames are listed in the Table I.

\section{A. Kinematic Analysis}

Considering the vector loops O-A-B-C-D-O and O-A-B$\mathrm{O}$ shown in Fig. 2(b), the loop closure equations in form of complex members are: (a)

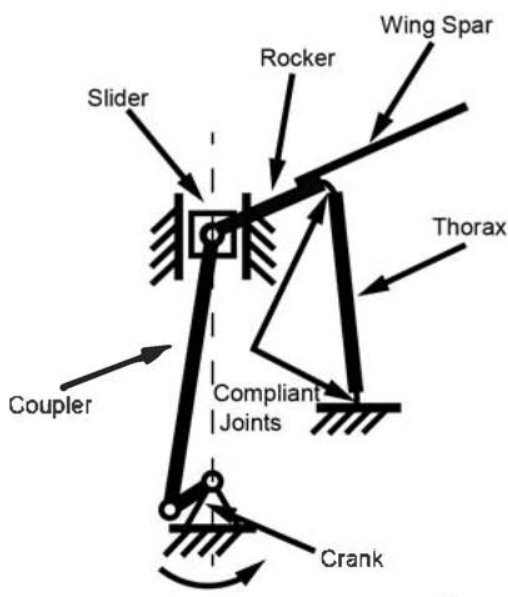

(b)

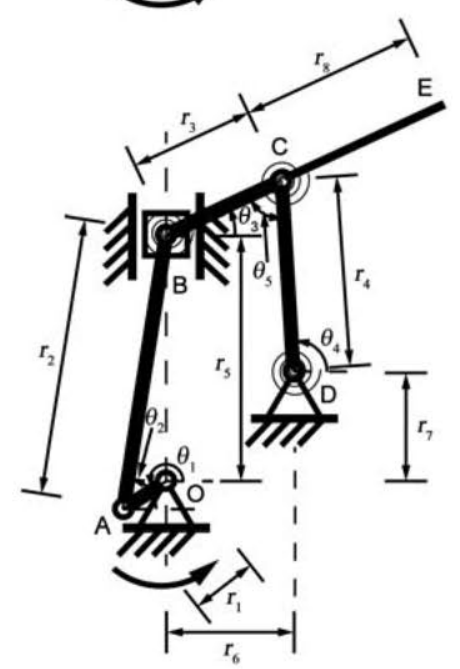

Fig. 2. (a) A schematic view of the compliant transmission mechanism during upstroke. (b) Pseudo-rigid-body model of the compliant transmission mechanism.

$$
\begin{gathered}
r_{1} e^{j \theta_{1}}+r_{2} e^{j \theta_{2}}-r_{3} e^{j\left(\pi+\theta_{3}\right)}-r_{4} e^{j \theta_{4}}-r_{6} e^{j 0}-r_{7} e^{j \frac{\pi}{2}}=0 \\
r_{1} e^{j \theta_{1}}+r_{2} e^{j \theta_{2}}-r_{5} e^{j \frac{\pi}{2}}=0
\end{gathered}
$$

where $r_{i}(i=1,2, \ldots, 6,7)$ are lengths of links and $\theta_{j}(j=$ $1,2, \ldots, 4)$ are angular positions of the corresponding links.

Since this driving mechanism has only one degree of freedom (DoF), the angular position of the crank $\theta_{1}$, thus the rest angular positions $\theta_{2}, \theta_{3}, \theta_{4}$ can be treated as functions in terms of $\theta_{1}$. Here we define $\dot{\theta}_{i}=\frac{\mathrm{d} \theta_{i}}{\mathrm{~d} \theta_{1}}, \ddot{\theta}_{i}=\frac{\mathrm{d} \dot{\theta}_{i}}{\mathrm{~d} \theta_{1}}(i=1,2,3,4)$. Note that $\dot{\theta}_{1}$ equals 1 and $\ddot{\theta}_{1}$ equals 0 , since the motor is considered to be rotating at constant speed, whose value is 600 rpm. Considering the velocity and acceleration of the angular position $\theta_{i}(i=1,2,3,4)$ with respect to time $t, \omega_{i}$ and $\alpha_{i}$, which are defined as $\omega_{i}=\frac{\mathrm{d} \theta_{i}}{\mathrm{~d} t}, \alpha_{i}=\frac{\mathrm{d} \omega_{i}}{\mathrm{~d} t}$, therefore, the velocity $\omega_{i}$ and acceleration $\alpha_{i}$ can be expressed as $\omega_{i}=\dot{\theta}_{i} \omega_{1}$ and $\alpha_{i}=\ddot{\theta}_{i} \omega_{1}^{2}+\dot{\theta}_{i} \alpha_{1}$. 


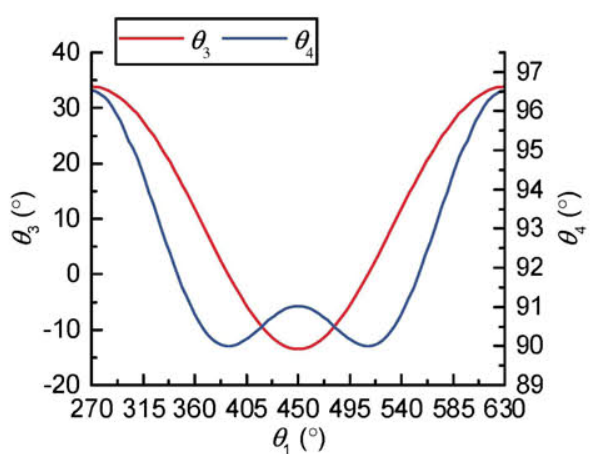

Fig. 3. Variations on the flapping angle of a wing and the torsional angle at compliant joint D during a flapping cycle.

When the initial values of angular positions $\theta_{1}, \theta_{2}, \theta_{3}, \theta_{4}$ are known, their corresponding values in a whole flapping cycle can be solved after separating real and imaginary components of equations (1) (2) and combining them together. Fig. 3 shows the flapping angle $\theta_{3}$ and the angular variety $\theta_{4}$ of the thorax during a flapping cycle. Similarly, angular velocities $\dot{\theta}_{i}$ and $\omega_{i}$ and accelerations $\alpha_{i}(i=2,3,4)$ of the links can also be computed according to the first and second order derivatives of the two position equations (1) and (2).

\section{AERODYNAMIC TORQUE}

Aerodynamic torques generated by flapping wings play a very important role in the stable flights of FWAVs. In this section, we analyse the aerodynamic torque produced by wing motions in a cycle. To this purpose, an aerodynamic model based on the blade element theory [19] was employed. For simplifying the calculation, the irregular shape of a wing is approximated with a rectangle, whose length and width equal the length of the wing spar $r_{8}$ and the average wing chord $c$, respectively. Note that the average chord $c$ is computed according to wings' equivalent surface area. Here $c$ equals $10.25 \mathrm{~cm}$. The wing is assumed to be rigid, i.e., without any twist and bend along the wing chord and the leading edge. The mass of the wing is considered to be negligible. Therefore, the normal force produced by a single blade can be expressed according to the theory in [19] as follows:

$$
d F_{a}=-\frac{1}{2} \rho C_{1}|V(r, t)|^{2} \operatorname{sgn}(V(r, t)) c d r,
$$

where $V(r, t)$ is the absolute velocity of an element, whose direction is contrary to the motion of the wing, $\rho$ is the air density, $1.23 \mathrm{~kg} / \mathrm{m}^{3}$, and $C_{1}$ is the normal force coefficient of the blade [20]. Note that $V(r, t)$ consists of two parts: translational velocity $V(r, t)_{T}$ and rotational velocity of wings $V(r, t)_{R}$ (see Fig. 4). Thus, $V(r, t)=V(r, t)_{T}+V(r, t)_{R}$ and $|V(r, t)|=\left|V(r, t)_{T}\right|+\left|V(r, t)_{R}\right|$. Be ware that $\left|V(r, t)_{T}\right|=$ $r_{4} \omega_{4} \cos \left(\theta_{5}\right),\left|V(r, t)_{R}\right|=r \omega_{3}$. Here, $\theta_{5}$ is the intersection angle between the rocker $r_{3}$ and the thorax $r_{4}$ as shown in Fig. 4. As the value of the translation velocity $V(r, t)_{T}$ is relatively small, its effect of wings' movements is also relatively few.

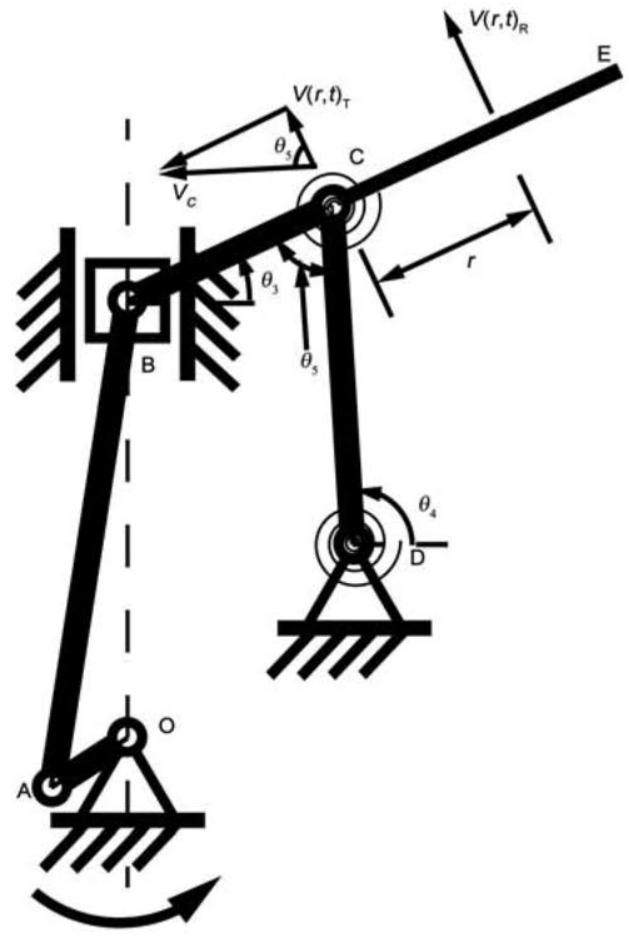

Fig. 4. The sketch of the velocity analysis on the wing.

Therefore, $V(r, t)$ and $V(r, t)_{R}$ can be treated as they have the same values, i.e., $V(r, t)=V(r, t)_{R}, \operatorname{sgn}(V(r, t))=\operatorname{sgn}\left(V(r, t)_{R}\right)$. Since $V(r, t)_{R}$ is positively proportional to $\omega_{3}, \operatorname{sgn}(V(r, t))$ can be described in terms of $\operatorname{sgn}\left(\omega_{3}\right)$ as $\operatorname{sgn}(V(r, t))=\operatorname{sgn}\left(\omega_{3}\right)$.

The instantaneous aerodynamic torque $\tau_{a}$ can be expressed by the instantaneous force $d F_{a}$ times the distance $r$ along the wing spar,

$$
d \tau_{a}=-\frac{1}{2} \rho C_{1} r^{3} \omega_{3}^{2} \operatorname{sgn}\left(\omega_{3}\right) c d r
$$

\section{InPut TORQue CALCULATION BASED ON THE PRINCIPLE OF VIRTUAL WORK}

After the kinematic analysis of the mechanism and calculation of aerodynamic torque produced by wings, we will derive the input torque required from the motor on the basis of the principle of virtual work. This method is well suitable for PRB models since it treats the system as a whole, and internal interaction forces are not needed to be considered. Furthermore, elastic potential energy stored in the compliant joints can be easily taken into account. Here, the transmission system is supposed to work in a plane. The masses of links (except the wing spar), inertial forces and frictions at joints are negligible in comparison with external aerodynamic forces and applied torque from the motor.

According to the PRB model shown in the Fig. 2, the generalized coordinate is chosen to be the angular displacement of the crank $\theta_{1}$ and its virtual displacement is $\delta \theta_{1}$. The total virtual work $\delta W$ includes three parts: the work done by the motor through external input torque $\tau_{m}, \delta W_{m}$, the 
work caused by inertial and aerodynamic torques of the wing, $\delta W_{w}=\delta W_{\text {inert }}+\delta W_{w}$, and the work stored by the equivalent springs at compliant joints, $\delta W_{s}$. Thus,

$$
\delta W=\delta W_{m}-\delta W_{w}-\delta W_{s}
$$

Since the mass of wing membrane is relatively small, the mass of wing approximately equals the mass of wing spar. As shown in the Fig. 2, the motion of wing spar can be treated as a synthesis of a axis-fixed rotational movement around the joint $\mathrm{C}$ and a translational movement around the joint $\mathrm{D}$. Therefore, the virtual work produced by the inertial torque of the rectangular wing is simply given as

$$
\delta W_{\text {inert }}=\frac{1}{3} m_{8} r_{8}^{2} \alpha_{3} \delta \theta_{3}+m_{8} r_{4}^{2} \alpha_{4} \delta \theta_{4}
$$

where $m_{8}$ is the mass of the wing spar. $\frac{1}{3} m_{8} r_{8}^{2}$ and $m_{8} r_{4}^{2}$ are the inertias of the wing with respect to the joint $\mathrm{C}$ and $\mathrm{D}$, respectively. In addition, the virtual work $\delta W_{a}$ caused by aerodynamic forces is given as $\delta W_{a}=-\tau_{a} \delta \theta_{3}$, therefore,

$$
\delta W_{w}=\frac{1}{3} m_{8} r_{8}^{2} \alpha_{3} \delta \theta_{3}+m_{8} r_{4}^{2} \alpha_{4} \delta \theta_{4}-\tau_{a} \delta \theta_{3}
$$

The virtual work at compliant joints is $\delta W_{s}=-\sum_{i=1}^{2} T_{i} \delta \psi_{i}$, where $T_{i}=-K_{i} \psi_{i},(i=1,2), K_{i}$ is the torsional stiffness constant at the $i$ th $(i=1,2)$ compliant joint. Note that that joints $\mathrm{C}, \mathrm{D}$ are defined as the 1 st and 2 nd joints, respectively. $\psi_{i}$ is the angular variation at the $i$ th joint, which is expressed as $\psi_{1}=\theta_{4}-\theta_{4,0}, \psi_{2}=\theta_{5}-\theta_{5,0}$ and those associated with $\delta \psi_{i}$ are: $\delta \psi_{1}=\delta \theta_{4}, \delta \psi_{2}=\delta \theta_{5}$. Here, $\theta_{j}, \theta_{j, 0}(j=4,5)$ are the angular position and neutral angular position at the two compliant joints, respectively. Be ware that $\theta_{5}=\theta_{4}-\theta_{3}, \theta_{5,0}=$ $\theta_{4,0}-\theta_{3,0}$, therefore, $\delta \theta_{5}=\delta \theta_{4}-\delta \theta_{3}, \delta \psi_{2}=\delta \theta_{5}=\delta \theta_{4}-\delta \theta_{3}$. The virtual work stored at compliant joints is

$$
\begin{aligned}
\delta W_{s}= & K_{1}\left(\theta_{4}-\theta_{40}\right) \delta \theta_{4} \\
& +K_{2}\left[\left(\theta_{4}-\theta_{3}\right)-\left(\theta_{4,0}-\theta_{3,0}\right)\right]\left(\delta \theta_{4}-\delta \theta_{3}\right)
\end{aligned}
$$

According to the principle of the virtual work, the total virtual work is set to equal to zero. The equation (5) can be solved in terms of the unknown input torque $\tau_{m}$,

$$
\begin{aligned}
\tau_{m}= & \frac{1}{3} m_{8} r_{8}^{2} \alpha_{3} \dot{\theta}_{3}+m_{8} r_{4}^{2} \alpha_{4} \dot{\theta}_{4}-\tau_{a} \dot{\theta}_{3}+K_{1}\left(\theta_{4}-\theta_{40}\right) \dot{\theta}_{4} \\
& +K_{2}\left[\left(\theta_{4}-\theta_{3}\right)-\left(\theta_{4,0}-\theta_{3,0}\right)\right]\left(\dot{\theta}_{4}-\dot{\theta}_{3}\right)
\end{aligned}
$$

\section{Optimization DESIGN}

It can be found from the equation (9) that the primary attributes, including the torsional stiffness of the virtual spring at the compliant joint $K_{i}(i=1,2)$ and neutral angular position $\theta_{j, 0}(j=3,4)$, influence the input torque from the motor. The effect of each attribute on the input torque is shown in Fig. 5 and 6, respectively. As for the Fig. 5 (Top), the peak torques over a cycle fall down when the stiffness at compliant joint C $K_{1}$ increases from 0 (pure rigid-body mechanism, RBM) to $0.6 \mathrm{Nm} / \mathrm{rad}$ and then the values rise from 0.6 to $1 \mathrm{Nm} / \mathrm{rad}$. The
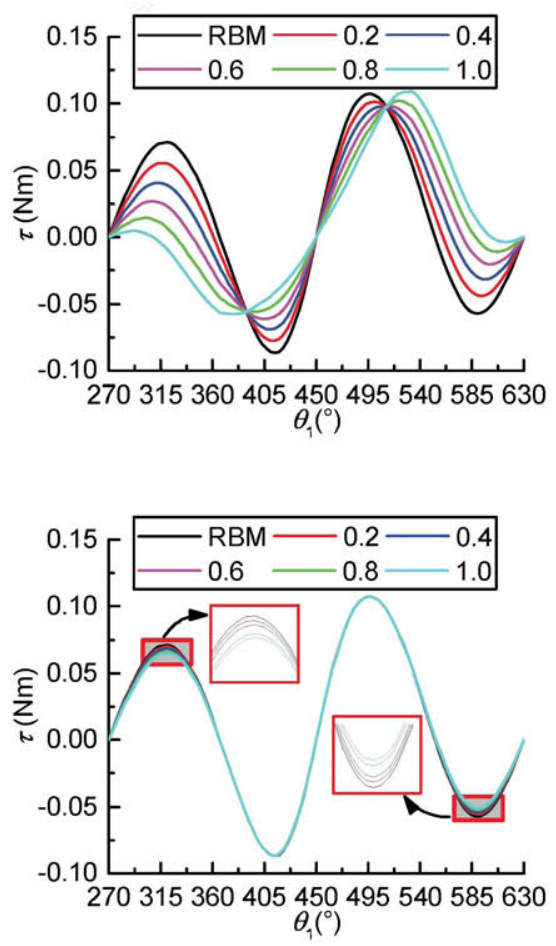

Fig. 5. Variation of the input torque from the motor in function of the torsional stiffness of virtual springs at joint $\mathrm{C}$ and D. Top: $K_{1}=0$ (RBM), 0.2, 0.4, $0.6,0.8,1 \mathrm{Nm} / \mathrm{rad}$ while $K_{2}=0 \mathrm{Nm} / \mathrm{rad}$; Bottom: $K_{2}=0$ (RBM), 0.2, 0.4 , $0.6,0.8,1 \mathrm{Nm} / \mathrm{rad}$ whereas $K_{1}=0 \mathrm{Nm} / \mathrm{rad}$. In both cases, $\theta_{3.0}$ and $\theta_{4.0}$ equal $0^{\circ}$ and $90^{\circ}$, respectively.

value of the peak torque is the minimum when $K_{1}$ equals 0.6 $\mathrm{Nm} / \mathrm{rad}$. The value of the trough torque always increases when $K_{1}$ varies from 0 to $0.8 \mathrm{Nm} / \mathrm{rad}$ and then slightly decreases when $K_{1}=1 \mathrm{Nm} / \mathrm{rad}$. From the Fig. 5 (Bottom), it is clear that the maximum and minimum values of the input torque seem to be the same with the variation of the stiffness at joint $\mathrm{D} K_{2}$ from 0 to $1 \mathrm{Nm} / \mathrm{rad}$ with a increment $0.2 \mathrm{Nm} / \mathrm{rad}$. Comparing with the pure rigid-body mechanism (RBM), the peak torques during the downstroke (from $270^{\circ}$ to $450^{\circ}$ ) slightly decrease while the values at the trough points during the upstroke (from $450^{\circ}$ to $630^{\circ}$ ) lightly increase with the rise of the stiffness at joint D.

In the Fig. 6 (Top), the peak torque in a cycle is the minimum for the compliant mechanism when the value of $\theta_{3,0}$ is chosen to be $10.5^{\circ}$. The minimum value is smaller than that of a RBM. While $\theta_{3,0}$ varies from $-1.5^{\circ}$ to $33.9^{\circ}$, none of the peak torque exceed the maximum torque needed for a RBM. From Fig. 6 (bottom), it can be seen that the peak torque during a whole flapping period is minimum when the neutral angle at joint $\mathrm{D} \theta_{4,0}$ equals $\pi / 2$. With the rise of the angle $\theta_{4.0}$, the absolute values at the peak and trough points increase, which clearly violates the objective of minimizing the peak torque and thereby lowering the shock of the motor. When the values of $\theta_{4,0}$ are bigger than $93^{\circ}$, the maximum input torque required for a compliant mechanism are even greater 

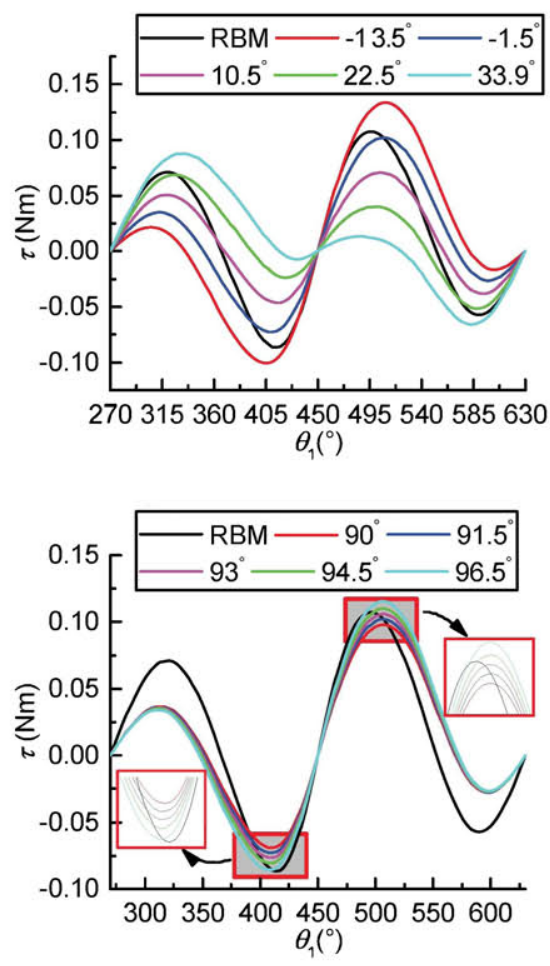

Fig. 6. Variation of the input torque from the motor in function of the neutra angular positions at joint $C$ and $\mathrm{D}$. Top: $\mathrm{RBM}, \theta_{3,0}=-13.5^{\circ},-1.5^{\circ}, 10.5^{\circ}$, $22.5^{\circ}, 33.9^{\circ}$ while $\theta_{4,0}=90^{\circ}$; Bottom: RBM, $\theta_{4,0}=90^{\circ}, 91.5^{\circ}, 93^{\circ}, 94.5^{\circ}$, $96.5^{\circ}$ while $\theta_{3,0}=0^{\circ}$. In both cases, $K_{1}$ and $K_{2}$ equal 0.4 and $0.6 \mathrm{Nm} / \mathrm{rad}$ respectively.

than the corresponding value for a RBM, which suggests that choosing a proper angular position of $\theta_{4,0}$ is important in order to minimize the peak torque. Thus, for the purpose of reducing the torque required as possible, the optimization of $K_{1}, K_{2}$, $\theta_{3,0}$ and $\theta_{4,0}$ is the key. Furthermore, to make sure the peak torques $\tau_{m_{\max }}$ and $\tau_{m_{\min }}$ minimize as possible and meanwhile the input torque varies smoothly over a whole flapping cycle, the objective function to optimization can be formulated as follows:

$$
F_{o b j}=\min \left(\tau_{m_{\max }}-\tau_{m_{\min }}\right)
$$

The corresponding constraint conditions are:

$$
K_{1} \in\left[K_{1 l}, K_{1 u}\right], K_{2} \in\left[K_{2 l}, K_{2 u}\right], \theta_{3,0} \in\left[\theta_{3 l}, \theta_{3 u}\right], \theta_{4,0} \in\left[\theta_{4 l}, \theta_{4 u}\right]
$$

where $\tau_{m_{\max }}$ and $\tau_{m_{\min }}$ are the maximum and minimum values of input torque over a flapping cycle, respectively. $K_{i l}$, $K_{i u}(i=1,2), \theta_{j, l}, \theta_{j, u}(j=3,4)$ are the lower and upper bounds of $K_{i}(i=1,2), \theta_{j, 0}(j=3,4)$, respectively. The situation with four constraints are referred to as Case $I$ in the following. According to the analysis on the four primary attributes in previous section, those bound parameters used for the optimization can be determined for the case at hand as shown in Table II. Note that the lower bound of $\theta_{4,0}$ is limited to be $90^{\circ}$ due to geometrical constraints. Meanwhile, the values
TABLE II

PARAMETERS USED FOR DESIGN OPTIMISATION

\begin{tabular}{c||c||c}
\hline Parameter & Lower Bound & Upper Bound \\
\hline \hline$K_{1}(\mathrm{Nm} / \mathrm{rad})$ & 0 & 0.8 \\
\hline$K_{2}(\mathrm{Nm} / \mathrm{rad})$ & 0 & 1 \\
\hline$\theta_{3,0}\left({ }^{\circ}\right)$ & -1.5 & 33.9 \\
\hline$\theta_{4,0}\left({ }^{\circ}\right)$ & 90 & 93 \\
\hline
\end{tabular}

of $K_{i u}(i=1,2)$ are respectively set to be 0.8 and $1 \mathrm{Nm} / \mathrm{rad}$ according to the stiffness of materials used for flexible joints. In [17], the optimization problem is treated assuming that the two compliant joints have the same revolute stiffness and both their neutral angular positions are located in the middle of their motion ranges. Such assumptions can be expressed as,

$$
\begin{gathered}
K_{1} \in\left[K_{1 l}, K_{1 u}\right], K_{2} \in\left[K_{2 l}, K_{2 u}\right], K_{1}=K_{2} \\
\theta_{3,0}=\frac{1}{2}\left(\theta_{3_{\text {max }}}+\theta_{3_{\text {min }}}\right), \theta_{4,0}=\frac{1}{2}\left(\theta_{4_{\text {max }}}+\theta_{4_{\text {min }}}\right)
\end{gathered}
$$

where $\theta_{j_{\max }}, \theta_{j_{\min }}(j=3,4)$ are the upper and lower limits of the motion ranges with respect to $\theta_{j}$. Their values can be obtained in Fig. 3. This case is referred to as Case II in the following.

Objective function (10) is a single-objective optimization problem with constraint conditions. Here, we used a hybrid multi-swarm particle swarm optimization (HMPSO) algorithm [21] to solve the optimization problem. The results of the optimization process are in shown in Table III. According to the optimization results, several optimum values of the joint torsional stiffness and neutral angular position appear to stand at the bounds. This may indicate that, the values of the torsional stiffness at compliant joints have a significant influence on the input torque and the greater the valued of the stiffness are, the smaller the difference between the maximum and minimum input torque is. Furthermore, neutral positions at compliant joints tend to locate the centres of their respective angle variation ranges, which to some extend echo in concert with the assumptions on neutral positions in [17].

The input torques required for a rigid-body mechanism and compliant transmission mechanisms with different limitations (CTM Cases I and II) are shown in Fig. 7. As it can be noticed, both the mechanisms employing compliant joints with well-tuned parameters can reduce the peak to torque required from the motor comparing to the counterpart of the fully rigid-body mechanism (labelled RBM). Specifically, the maximum input torque for CTM I is reduced by $66.0 \%$ and the minimum torque is decreased by $71.7 \%$ compared to the RBM. Similarly, for CTM II, the input torque relative to the pure $\mathrm{RMB}$, is reduced by $40.4 \%$ and $73.9 \%$, for maximum and minimum input torques, respectively. In addition, it can also be found that the peak torque optimized of the compliant mechanism obtained by using the limitations Case I is smaller that the corresponding gotten by utilizing the constraints Case II, which shows that the constraint method provided in this 
TABLE III

OPTIMISATION RESULTS FOR TWO KINDS OF CONSTRAINT CONDITIONS

\begin{tabular}{c||c||c||c||c}
\hline Condition & $K_{1}(\mathbf{N m} / \mathbf{r a d})$ & $K_{2}(\mathbf{N m} / \mathbf{r a d})$ & $\theta_{3,0}\left({ }^{\circ}\right)$ & $\theta_{4,0}\left({ }^{\circ}\right)$ \\
\hline \hline Case I & 0.8 & 1 & 12.2 & 90 \\
\hline Case II & 0.8 & 0.8 & 10.2 & 93.3 \\
\hline
\end{tabular}

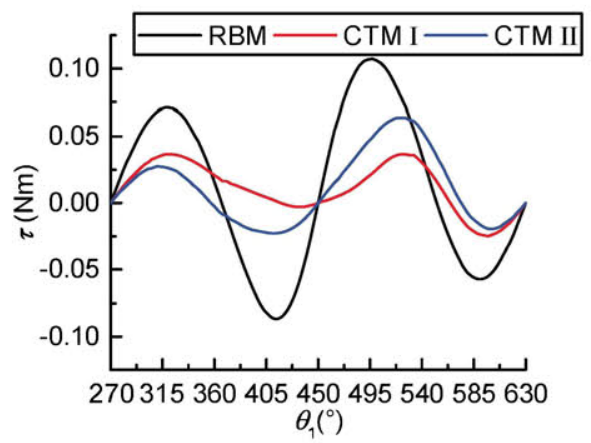

Fig. 7. Input torque required of the flapping mechanism with three differen transmission systems: Rigid-body Mechanism (RBM); Compliant Transmission Mechanism with constraints Case I (CTM I); Compliant Transmission Mechanism with constraints Case II (CTM II).

paper is better than that used in [17]. In fact, the constraint conditions Case II is only a specific subset of the limitations Case I.

\section{CONCLUSIONS}

In this paper, we have presented a compliant transmission mechanism with compliant hinges for a flapping-wing aerial vehicle. The compliant hinges can be treated as revolute joints with virtual torsional springs. The potential energies stored and released influence the input torque required from the motor, which lowers the shocks to the motor.

A virtual-work-based method was adopted to analyse the input torque needed by a rotational motor to produce the flapping movements. Following the analysis, the optimization design of compliant joints in terms of the stiffness of virtual spring and the initial angular position has been carried out. The results of the optimization show that, theoretically, up to $66.0 \%$ of the maximum input torque, and up to $73.9 \%$ of the minimum torque could be reduced compared to the full rigid-body mechanism. The results obtained demonstrate that the compliant mechanism with proper design can significantly reduce the peak input torque and avoid the sharp shocks of the driving motor. Future work will be devoted to the physical instantiation of the mechanism using the optimized theoretical values for the key parameters obtained in this study.

\section{ACKNOWLEDGMENT}

The work of the first and third authors have been sponsored by the China Scholarship Council(CSC). This work has been co-funded by the RoboCity2030-III-CM project (Robotica aplicada a la mejora de la calidad de vida de los ciudadanos. fase III; S2013/MIT-2748), funded by Programas de Actividades I+D en la Comunidad de Madrid and co-funded by Structural Funds of the EU.

\section{REFERENCES}

[1] G. Pratt and M. Williamson, "Elastic actuator for precise force control," 1997, uS Patent 5,650,704.

[2] R. Madangopal, Z. Khan, and S. Agrawal, "Biologically inspired design of small flapping wing air vehicles using four-bar mechanisms and quasisteady aerodynamics," Journal of Mechanical Design, vol. 127, no. 4 pp. 809-816, 2005.

[3] S. Baek, K. Ma, and R. Fearing, "Efficient resonant drive of flappingwing robots," in 2009 IEEE/RSJ International Conference on Intelligent Robots and Systems (IROS), pp. 2854-2860, 2009.

[4] L. Hines, D. Campolo, and M. Sitti, "Liftoff of a motor-driven, flappingwing microaerial vehicle capable of resonance," IEEE Transactions on Robotics, vol. 30, no. 1, pp. 220-232, 2014.

[5] M. Azhar, D. Campolo, G. Lau, L. Hines, and M. Sitti, "Flapping wings via direct-driving by dc motors," in 2013 IEEE International Conference on Robotics and Automation (ICRA), pp. 1397-1402, 2013.

[6] G. Lau, Y. Chin, J. T. Goh, and R. Wood, "Dipteran-insect-inspired thoracic mechanism with nonlinear stiffness to save inertial power of flapping-wing flight," IEEE Transactions on Robotics, vol. 30, no. 5, pp. 1187-1197, 2014.

[7] R. Wood, E. Steltz, and R. Fearing, "Optimal energy density piezoelectric bending actuators," Sensors and Actuators A: Physical, vol. 119, no. 2, pp. 476-488, 2005.

[8] M. Sitti, "Piezoelectrically actuated four-bar mechanism with two flexible links for micromechanical flying insect thorax," IEEE/ASMF Transactions on Mechatronics, vol. 8, no. 1, pp. 26-36, 2003.

[9] B. Cheng, J. Roll, X. Deng et al., "Modeling and optimization of an electromagnetic actuator for flapping wing micro air vehicle," in 2013 IEEE International Conference on Robotics and Automation (ICRA), pp. 4035-4041, 2013.

[10] J. Roll, B. Cheng, X. Deng et al., "An electromagnetic actuator for high-frequency flapping-wing microair vehicles," IEEE Transactions on Robotics, vol. 31, no. 2, pp. 400-414, 2015

[11] J. Roll, B. Cheng, and X. Deng, "Design, fabrication, and experiments of an electromagnetic actuator for flapping wing micro air vehicles," in IEEE International Conference on Robotics and Automation (ICRA), pp. $809-815,2013$.

[12] T. Tantanawat and S. Kota, "Design of compliant mechanisms for minimizing input power in dynamic applications," Journal of Mechanical Design, vol. 129, no. 10, pp. 1064-1075, 2007.

[13] R. Wood, "Liftoff of a $60 \mathrm{mg}$ flapping-wing mav," in IEEE/RSJ International Conference on Intelligent Robots and Systems (IROS), pp. 1889. 1894, 2007.

[14] A. Cox, D. Monopoli, D. Cveticanin, M. Goldfarb, and E. Garcia, "The development of elastodynamic components for piezoelectrically actuated flapping micro-air vehicles," Journal of Intelligent Material Systems and Structures, vol. 13, no. 9, pp. 611-615, 2002.

[15] K. Mateti, R. Byrne-Dugan, C. D. Rahn, S. Tadigadapa et al., "Monolithic suex flapping wing mechanisms for pico air vehicle applications," Journal of Microelectromechanical Systems, vol. 22, no. 3, pp. 527-535, 2013.

[16] Y.-W. Chin and G.-K. Lau, "clicking compliant mechanism for flappingwing micro aerial vehicle," in IEEE/RSJ International Conference on Intelligent Robots and Systems (IROS), pp. 126-131, 2012.

[17] R. Sahai, K. Galloway, and R. Wood, "Elastic element integration for improved flapping-wing micro air vehicle performance," IEEE Transactions on Robotics, vol. 29, no. 1, pp. 32-41, 2013.

[18] L. L. Howell, Compliant mechanisms, 2001.

[19] C. Ellington, "The aerodynamics of hovering insect flight. i. the quasisteady analysis," Philosophical Transactions of the Royal Society B: Biological Sciences, vol. 305, no. 1122, pp. 1-15, 1984.

[20] Z. Khan, S. Agrawal et al., "Design of flapping mechanisms based on transverse bending phenomena in insects," in 2006 IEEE International Conference on Robotics and Automation (ICRA), pp. 2323-2328, 2006.

[21] Y. Wang and Z. Cai, "A hybrid multi-swarm particle swarm optimization to solve constrained optimization problems," Frontiers of Computer Science in China, vol. 3, no. 1, pp. 38-52, 2009. 